\title{
ASPECTS OF TWO WAY SHAPE MEMORY IN NITI-SILICONE COMPOSITE MATERIALS
}

\author{
K. ESCHER and E. HORNBOGEN \\ Ruhr-Universität Bochum, Institut für Werkstoffe, Postfach 1021 48, D-4630 Bochum, Germany
}

\begin{abstract}
The influence of a silicone layer on the two way effect (TWE) of an NiTi composite material is investigated. Stresses stored in the silicone during the heating process favour the reverse shape change during the cooling process. An improvement of the TWE compared with the plain metallic material is measured. The amount depends on different variables.
\end{abstract}

\section{Introduction}

Different ways are possible to achieve a two way effect of a shape memory element $/ 1 /$. The intrinsic effect of the plain metallic material leads to an easy structure of a one component element. An external force, e. g. a spring, allows an accurate and reliable reverse shape change ("TWE in system"). Both can be combined if rubber is used as second component of a shape memory composite material instead of the external spring. The first shape change creates deformation stresses inside the rubber. The stresses support the reverse shape change during the cooling process. Consequently, an improved two way effect of a one component element can be expected compared to the effect of the plain metallic material.

Only a few publications were engaged in this subject matter so far $/ 2,3 /$. A comprehensive investigation has not yet been carried out. All measurements were taken by composite materials consisting of an SMA and silicone as second component. Silicones are appropriate because of their capacity to preserve the elastic properties up to temperatures of $200{ }^{\circ} \mathrm{C}$. They exhibit an elongation at tear between $90 \%$ and $600 \%$. Silicones are excellent electrical insulators which is important if the shape memory elements were to be warmed up by direct resistance heating.

\section{Theoretical Considerations}

For an improved two-way effect the reverse shape change during the cooling period has to be supported. This corresponds to the function mode of an actuator working with a biased spring. The shape memory element has to be pseudoplastically deformed in the martensitic state (Fig. 1). Afterwards the silicone has to be vulcanized on the surface of the SMA. Heating the as prepared elements leads to the shape change according to the austenite reverse transformation. Inside the silicone stresses are caused by this shape change. During the cooling process the phase transformation is influenced by these stresses. They favour the oriented formation of martensite. Smallest stresses may even affect the martensitic transformation.

The effect of the silicone layer on the TWE is characterized by a condition of equilibrium in the high temperature state:

$$
\mathrm{F}_{\mathrm{si}}=\mathrm{F}_{\mathrm{sma}}
$$

$\mathrm{F}_{\mathrm{si}}$ : force originated in the silicone.

$\mathrm{F}_{\mathrm{sma}}$ : force of the transforming component. 


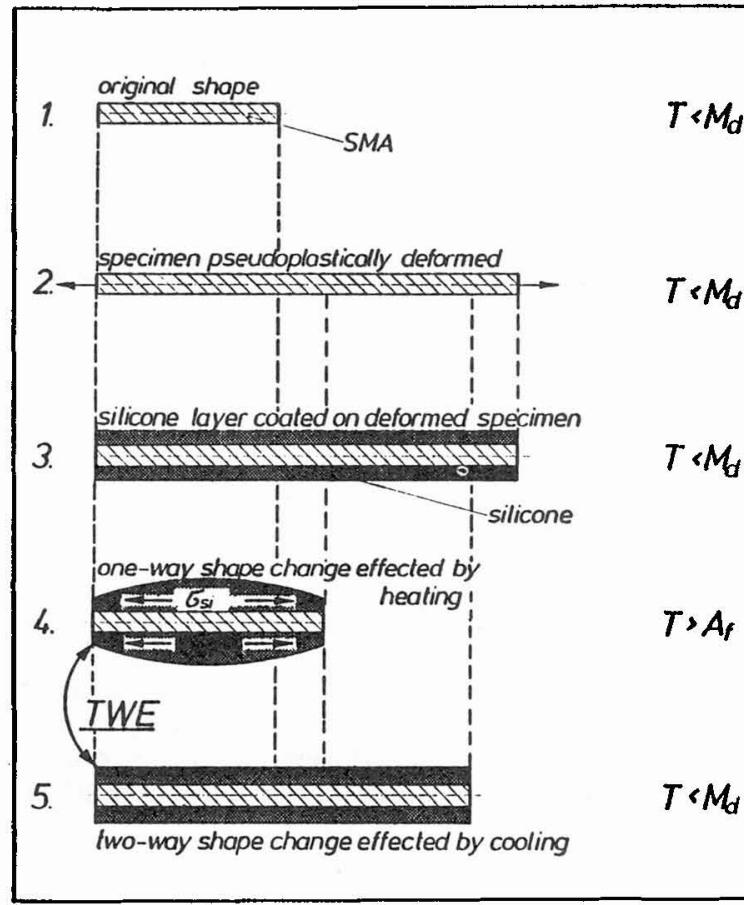

Fig. 1: Preparation of an SM-composite material with TWE, schematic, not in scale.

The forces can be verified by the following equations:

$$
\begin{aligned}
& \mathrm{F}_{\text {si }}=\Delta \mathrm{l} \cdot \mathrm{c}_{\mathrm{si}} \\
& \mathrm{F}_{\mathrm{sma}}=\mathrm{A} \cdot \sigma_{\mathrm{v}}
\end{aligned}
$$

The dependences derived by these equations are:

$\triangle \mathrm{l}$ : amount of deformation during the first shape change (one way effect (OWE))

$\mathrm{c}_{\mathrm{si}} \mathrm{i} \quad$ mechanical properties of the silicone (spring characteristic)

A. geometrical arrangement of the silicone (dimension, shape)

A: $\quad$ cross section of the SMA

$\sigma_{\mathrm{v}}$ : mechanical properties of the applied SMA amount of deformation of the first shape change (OWE)

Even smallest stresses can influence the two-way training. With increasing stress the training procedure becomes more and more unnecessary. If the ratio SMA/silicone is chosen in a suitable way no two-way training should have to be carried out for achieving a two-way effect. In case the relation is too big the shape change is expected to be impeded.

\section{Adhesion Tests}

Silicones can be classified in one-component and two-component types. One component silicones are usually used as leakproofing materials. They exhibit good bonding properties but need air humidity for curing. Therefore, isolated moulds for producing similar specimens cannot be used. Two-component silicones are more suitable for this kind of application. They do not react on atmospheric humidity and can be easily fabricated in teflon moulds. Two-component silicones are themselves often used as moulding material because of their good separation properties which is a disadvantage in case of the production of a composite material. A sufficient interfacial bond can be achieved with bonding agents only.

For proofing the best adhesion on NiTi four two-component silicones with different properties were investigated (Table 1). They were tested in a three point bending mode. The specimens were made from epoxy with two NiTi sheets, between which the silicones were placed. The 
surface of the SMA was either polished or chemically etched. The results were satisfying for three of the silicones which showed cohesive failure. The adhesion was poor for only one with adhesive failure. The etched surface of the SMA led to the best adhesion (Fig. 2). These results do not consider the deformation of the interface due to the two way shape change. Deformation of the interface can lead to laminar separation of the primer. Consequently, the most certain method to achieve a good adhesion even after a long period of cycling and shape change is to apply form closure.

Table 1: Properties of silicones investigated.

\begin{tabular}{|c|c|c|c|c|c|}
\hline $\begin{array}{l}\text { Two-Component } \\
\text { Silicones }\end{array}$ & $\begin{array}{c}\text { Type of } \\
\text { Polymerisation }\end{array}$ & $\begin{array}{l}\text { Shore } \\
\text { Hardness }\end{array}$ & $\begin{array}{c}\text { urs } \\
{\left[\mathrm{N} / \mathrm{mm}^{2}\right]}\end{array}$ & $\begin{array}{c}\begin{array}{c}\text { Elongation } \\
\text { at Tear }\end{array} \\
{[\%]}\end{array}$ & $\begin{array}{l}\text { Thermal } \\
\text { Conductivity } \\
\text { (T: 0-90\% }) \\
{\left[\frac{\mathrm{W}}{\mathrm{K} \cdot \mathrm{m}}\right]}\end{array}$ \\
\hline RTV-ME 428 & $\begin{array}{c}\text { Poly- } \\
\text { Condensation }\end{array}$ & $70 \pm 3$ & $6.0 \pm 0.5$ & $90 \pm 20$ & 0,24 \\
\hline RTV-M 533 & $\begin{array}{c}\text { Poly- } \\
\text { Condensation }\end{array}$ & $25 \pm 3$ & $5,0 \pm 0,5$ & $350 \pm 30$ & 0,21 \\
\hline RTV-ME 628 & Polyaddition & $47 \pm 5$ & $3,5 \pm 0,5$ & $230 \pm 30$ & 0,3 \\
\hline RTV-ME 646 & Polyaddition & $60 \pm 5$ & $5,5 \pm 0,5$ & $320 \pm 30$ & 0,35 \\
\hline
\end{tabular}

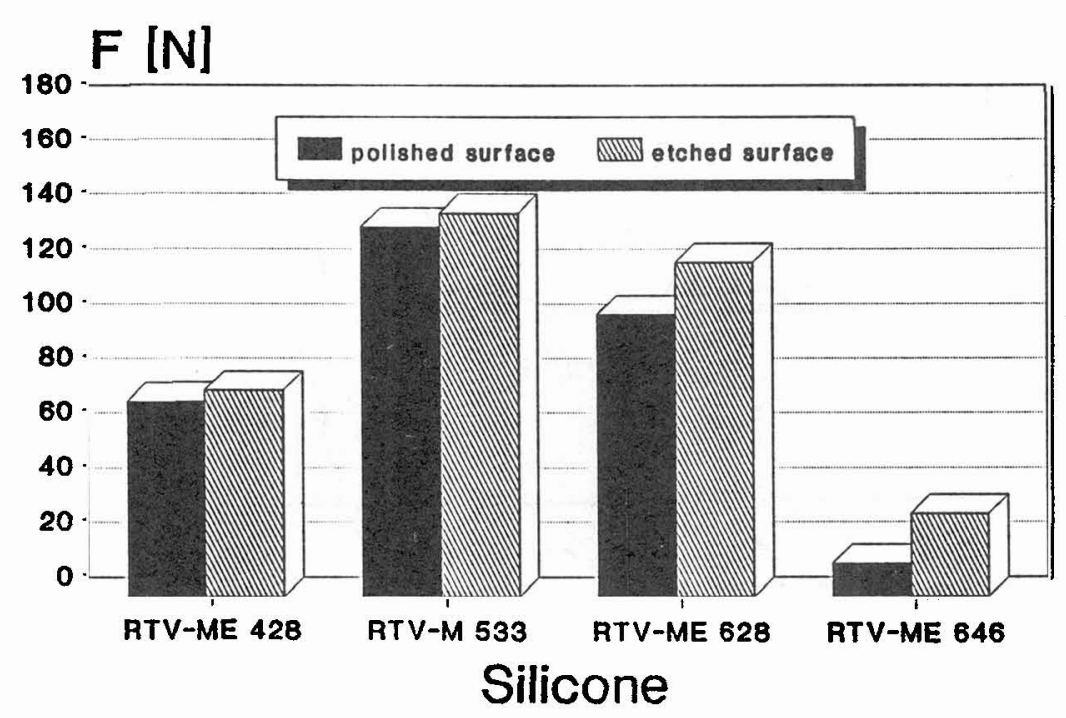

Fig. 2: Results of adhesion tests.

\section{Experimental methods}

Two different kinds of NiTi-silicone composite specimens were investigated with respect to their influence on the two-way effect: Tensile specimens and helical springs (Fig. 3). Both were tested as plain metallic material or coated with a silicone layer. An NiTi-alloy was used with transformation temperatures above room temperature. On the surface of the tensile specimens a profile was fissured to realize the form closure. After fabricating the specimens were annealed for 1 hour at $600{ }^{\circ} \mathrm{C}$ to reduce internal stresses. They were etched in aqua regia with additions of ammonium hydrogen fluoride to eliminate the oxide skin and enlarge the surface. After that procedure the specimens were deformed at room temperature for different fixed amounts in a tensile testing machine. The as deformed specimens were first coated with a primer and secondly with a silicone layer of RTV-ME 628 . The outer diameter of the specimens was $6 \mathrm{~mm}$. 


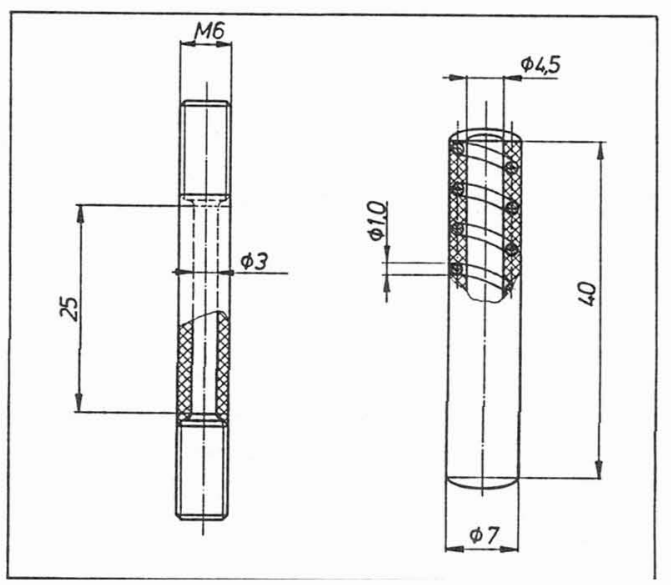

Fig. 3: Specimens used with silicone as second component:

The as prepared specimens were trained in a tensile testing machine, starting with the heating process (Fig. 4). Twenty thermo--mechanical cycles were carried out consisting of deformation, unloading, heating and cooling. The training was conducted for particular amounts of shape change $\epsilon$. The effect was trained into longitudinal direction. When the training process was finished the threads of the investigated specimens were removed and the residual parts were investigated by dilatometric measurements.

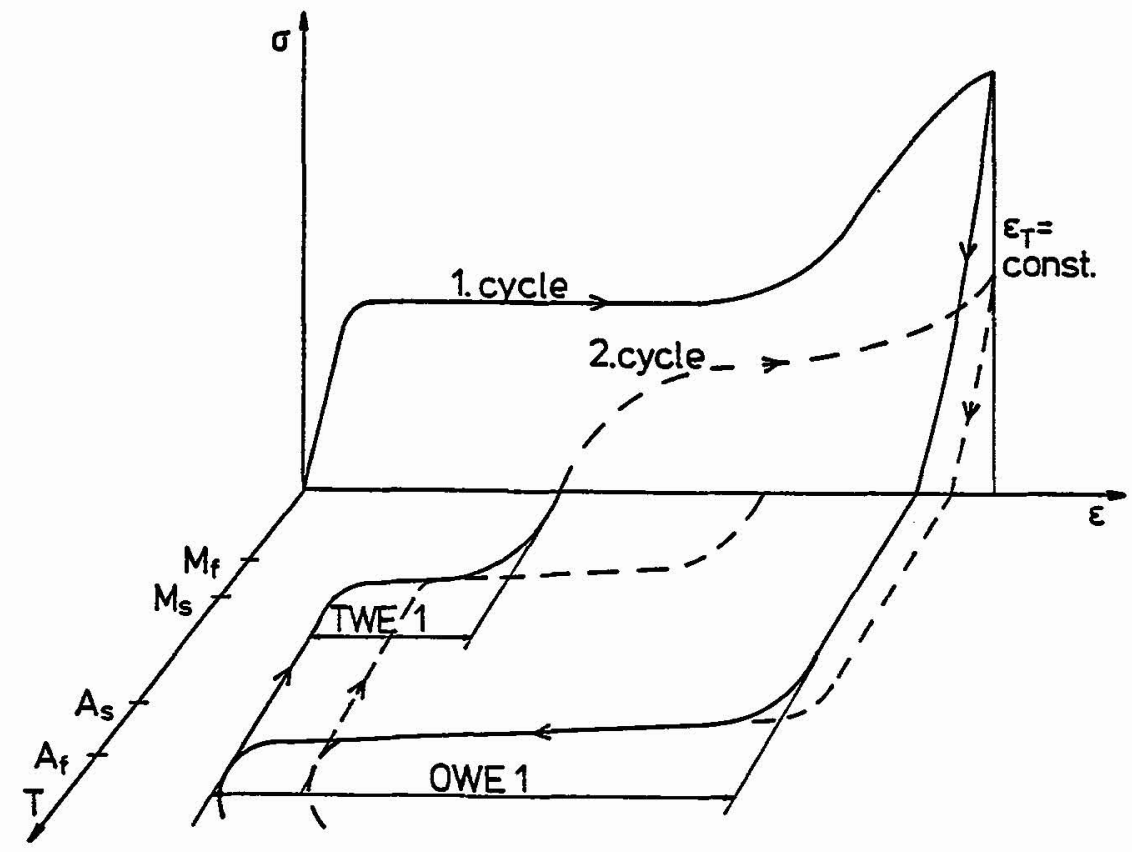

Fig. 4: Thermo-mechanical training process, schematic.

The helical springs were coiled with a total length of $12 \mathrm{~mm}$ and deformed to $40 \mathrm{~mm}$. After the deformation the surface was coated with a primer and the silicone was deposited on the whole spring. Heating led to the one-way shape change while the reverse shape change was effected by cooling. A training procedure was not necessary. Four different silicones were tested with the spring-element (Table 1). 


\section{Results and discussion}

In case of the tensile specimens the relation silicone-NiTi is too small to induce a TWE without training. A thermo-mechanical procedure is necessary but the TWE is improved by the elastomer. The influence of the silicone layer depends on the amount of deformation of the first shape change (Fig. 5). The stresses inside the silicone increase with an increasing amount of one-way shape change. Consequently the improvement of the TWE during the training procedure is low at small amounts of deformation but larger for high deformation rates (Fig. 6).
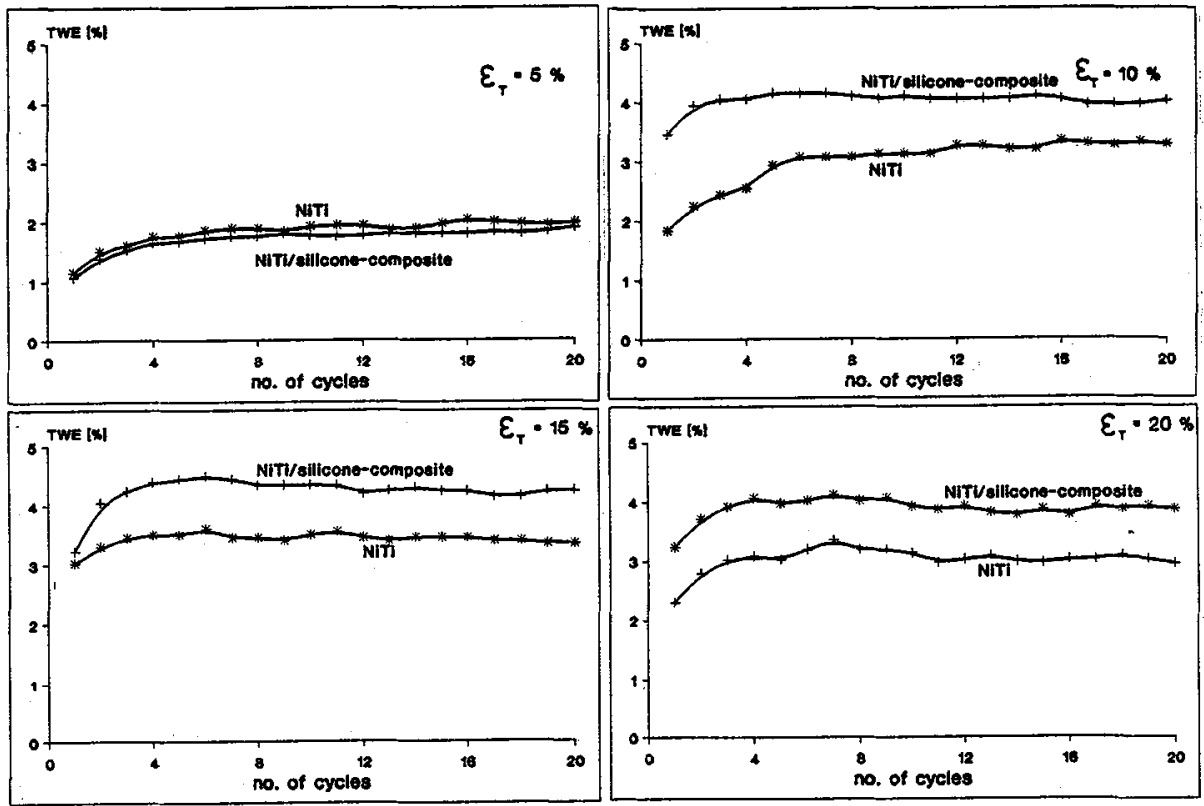

Fig. 5: TWE during the training process of SM-composite materials compared to plain metallic SMA. Different amounts of training strain $\epsilon_{\mathrm{T}}$.

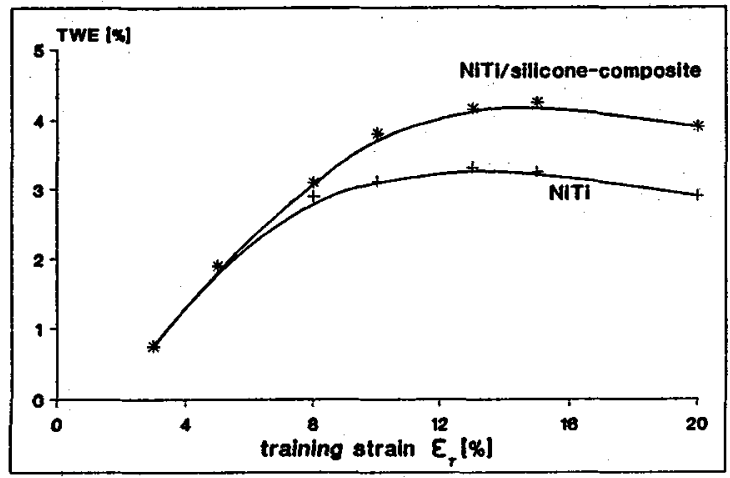

Fig. 6: Improved TWE of composite materials, depending on the training strain $\epsilon_{\mathrm{T}}$.

A TWE can be realized by the deformation stresses of the rubber only if the two components are chosen in an adequate relation. This can be shown by the second geometrical arrangement, the helical spring. The TWE already occurs after the first temperature change. A further advantage of the spring is the complete form closure. A laminar separation is impeded and a long lifetime can be expected. The amount of the effect depends on the chosen silicone as already described. The first shape change during the heating process is smaller with increasing hardness of the silicone (Fig. 7). The plain metallic material has the highest value while the composite material with Shore-Hardness 70 shows the smallest OWE. The TWE is characterized by the reverse shape 
change during the cooling process. The plain metallic material does not exhibit an effect. The composite-material with Shore-Hardness 25 produces the biggest TWE but this effect occurs for a shortened length. The OWE of this element is $20 \mathrm{~mm}$ which leads to a total length of $20 \mathrm{~mm}$ after the first shape change. The TWE during cooling leads to a reverse shape change of $9 \mathrm{~mm}$. Therefore the original length of $40 \mathrm{~mm}$ is not reached. This can be shown by the ratio TWE/OWE which is $100 \%$ for composites with higher amounts of Shore-Hardness orily (Fig. 8).
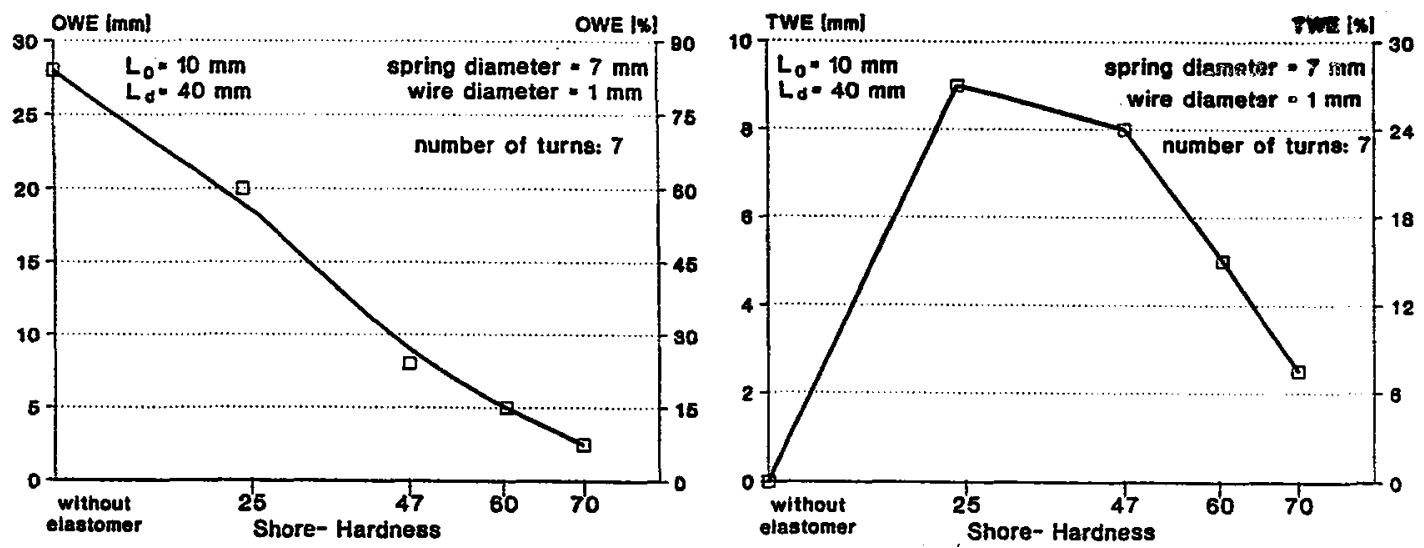

Fig. 7: SM-springs coated with different silicones; a) OWE, b) TWE.

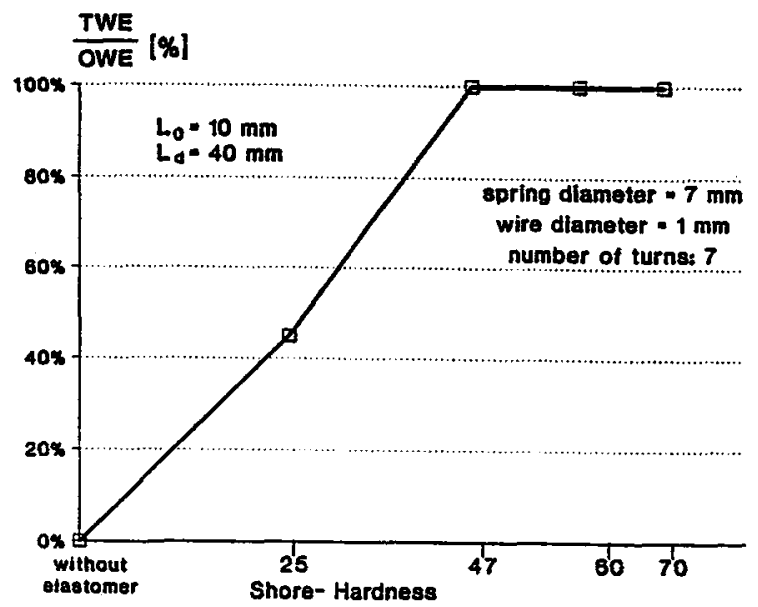

Fig. 8: Ratio of TWE and OWE according to the first shape change.

\section{Acknowledgement}

We gratefully acknowledge the support of the German Aerospace Agency (DARA) and the German Ministry for Research and Technology (BMFT 01 RS 88292).

\section{References}

/1/ K. Escher, E. Hornbogen, Robot-Grippers - An Application of Two-Way Shape Memory, Progress in Shape Memory, S. Eucken (ed.), DGM-Informationsges., Oberursel (1991), to be published.

/2/ M. Thumann, B. Velten, E. Hornbogen, Composites Containing Shape Memory Fibers,

/3/ K. Escher, K. Halter, E. Hornbogen, N. Jost, An Exploratory Study of Robotic Actuators With Shape Memory Alloys (SMA), Z. Flugwiss. Weltraumforsch. 14 (1990), 59-66. 